\title{
A Novel Method for Weighted Cooperative Spectrum Sensing in Cognitive Radio Networks
}

\author{
Xue JIANG \& Kunbao CAI \\ College of Communication Engineering, Chongqing University, Chongqing 400044, P. R. of China
}

\begin{abstract}
Considering that most existing techniques for weighted cooperative spectrum sensing have not comprehensively considered the influence of the space factor in wireless environment on the detection performance in cognitive radio systems, the authors of this paper present a novel method for improving the detection performance that combines the signal-to-noise ratio parameters and the path-loss parameters into the weights. The new technique includes the design of the formulas for weight assignment. Thus, the information of the signal-to-noise ratios and the path losses can be reasonably fused so that the mathematical model for enhancing detection performance is well established. Also, a spectrum sensing scenario in the wireless environment is simulated. By applying the randomly generated simulation parameters and their derived parameters, the simulation experiment is, respectively, performed in the environment of AWGN, Rayleigh and Nakagami wireless channels. The experimental results show that the new technique substantially improves the detection performance.
\end{abstract}

KEYWORD: Cognitive Radio Network; Cooperative spectrum sensing; Weighted cooperative; Energy detection; Path loss

\section{INTRODUCTION}

With the rapid development of wireless communication technology, the frequency spectrum is becoming more and more crowded. The spectrum resource has become the main bottleneck restricting the development of future wireless communications (Luo et al. 2009). In terms of, however, the present wireless communications, a considerable portion of the licensed users did not make full use of the fixed spectrum allocation, so the research, development and utilization of intelligent cognitive radio technology are expected to supply an effective way to solve such a problem in future wireless communications.

The cognitive radio with the help of the realtime sensing ability of white space can realize the dynamic management and allocation of the frequency spectrum, with the result that the spectrum resource utilization is expected to be improved greatly. Obviously, the spectrum sensing is one of the key technologies of cognitive radio systems, and is a premise and foundation to realize cognitive radio systems. At present, most portion of the spectrum bands have been allocated, so the challenge is how unlicensed users to share these bands without interferences with the licensed users (Luo et al.
2009, Lin et al. 2010).

The fundamental requirement of the spectrum sensing is that the detection time duration should be as short as possible, while having a higher detection probability and a lower missed or false alarm probability, so as to improve the spectrum utilization. The conventional Equal Gain Combining (abbreviated as EGC) is a typical method for the weighted cooperative spectrum sensing (Visser et al. 2008). This simple method assigns the same weight with unity to every Secondary User (abbreviated as SU). In other words, the EGC method did not consider the effect of the different SNRs received by individual SUs on the detection performance of the cognitive radio, which may leads a lower detection liability. Generally, the other weighted cooperative methods are the modification of the EGC method, which improve the detection performance by using different weight factors (Pan, et al. 2009, Visser et al. 2008, Zhou et al. 2007, Ghasemi et al. 2005). It is also noted that the spatial variation of the SUs has been considered in the weighted cooperative method (Shahid et al. 2008), where the weight coefficients determined by a ratio of logarithms.

On the basis of investigation of the above weighted cooperative algorithms, we present a novel cooperative spectrum sensing method that 
comprehensively utilizes the SNRs received by SUs and the path losses caused by the distance between the individual SUs and a Primary User (abbreviated as PU). The key point of the new method lies in the design of a reasonable parameter fusion scheme which will be introduced in this paper. By a simulated spectrum sensing scenario, the new method with a variety of simulation environment parameters is experimented in the AWGN channel, the Rayleigh and Nakagami fading channels. The experimental results show that the detection performance is substantially improved, while the implementation complexity is only slightly increased.

\section{SINGLE-NODE ENERGY DETECTION}

Under the condition of single node, the detection problem of an unknown deterministic signal in AWGN can be summarized into a binary hypothesis test as follows:

$$
y(t)=\left\{\begin{array}{cc}
n(t) & H_{0} \\
s(t)+n(t) & H_{1}
\end{array}\right.
$$

where $y(t)$ is the received signal by a SU, $s(t)$ is the transmitted signal by a PU, $n(t)$ is the AWGN with zero mean. Under the hypothesis $H_{1}$, the received signal $y(t)$ is the sum of the transmitted signal and the noise, i.e., the PU is using the present frequency band within the observation duration from 0 to $T$ seconds. Under the hypothesis $H_{0}$, the signal $y(t)$ consists only of the noise, that is, the frequency band is not being used. For the situation that only the working frequency band is known, but there is no any other prior knowledge of the deterministic signal transmitted by the PU, it is recognized that the energy detection method is perhaps the best choice for the above binary hypothesis test problem (Urkowitz, 1967, Digham, 2003). The basic structure of an energy detector is shown in Figure 1, where the ideal bandpass filter with bandwidth $W$ in $\mathrm{Hz}$ is used to filter out-ofband noise. The output $y_{o}(t)$ of the filter is then sent to the square law device whose output is integrated

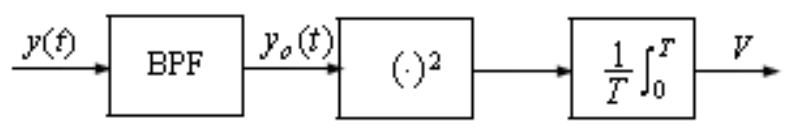

Figure 1. Energy detector

by the following integrator. The integrator output $V=\frac{1}{T} \int_{0}^{T} y_{o}^{2}(t) d t$ is just the average power of the filtered signal. The test statistic is selected as a normalized energy, $Y=\frac{1}{N_{0}} \int_{0}^{T} y_{o}^{2}(t) d t$, in which the quantity $N_{0}$ is the two-sided power spectral density of the noise. Then the test statistic for the energy detector can be represented as (Digham et al. 2003, Bin et al. 2008)

$$
Y=\left\{\begin{array}{cc}
\chi_{2 T W}^{2} & H_{0} \\
\chi_{2 T W}^{2}(2 \gamma) & H_{1}
\end{array}\right.
$$

where the time-bandwidth product $T W$ is assumed to be an integer value. This says that under the hypothesis $H_{0}$, the test statistic $Y$ is subject to a central $\chi^{2}$ distribution with $2 T W$ degrees of freedom; and under the hypothesis $H_{1}, Y$ follows a noncentral $\chi^{2}$ distribution with $2 T W$ degrees of freedom and a noncentral parameter $2 \gamma$. Here, $\gamma=E_{s} / 2 N_{0}$ is defined as the received SNR by the SU. Thus, in the AWGN channel, the detection and false-alarm probabilities are, respectively, given by

$P_{d}=P\left(Y>\lambda \mid H_{1}\right)=Q_{u}(\sqrt{2 \gamma}, \sqrt{\lambda})$

and

$P_{f}=P\left(Y>\lambda \mid H_{0}\right)=\Gamma(u, \lambda / 2) / \Gamma(u)$

where $u=T W, \lambda$ is the decision threshold, $Q_{u}(a, b)$ is the generalized Marcum $Q$-function, $\Gamma(\cdot)$ and $\Gamma(\cdot, \cdot)$ are the complete and incomplete gamma functions, respectively.

The average detection probability in a fading environment can be expressed as (Ghasemi et al. 2005, Zhou et al. 2007)

$\bar{P}_{d}=\int_{x}^{\infty} P_{d} f_{\gamma}(x) d x$

where $P_{d}$ is determined by (3), $f_{\gamma}(x)$ represents the probability density function of the SNR in different fading environments. Because $P_{f}$ is unrelated to the signal transmitted by the PU, it is fixed, as shown in (4).

\section{WEIGHTED COOPERATIVE METHOD}

\subsection{Basic Principle}

In the conventional method of the weighted cooperative spectrum sensing, each SU first detects the signal energy in the current band, and then sends a normalized energy value to a central Access Point (Access Point, AP). After receiving all of the normalized energy values, the AP calculates the weighted sum of these values in terms of a set of prescribed weights, and then compares the sum to a detection threshold to make a decision. Here the weighted sum can be represented as (Shahid et al. 2008) 


$$
Y_{w}=\sum_{i=1}^{N} W_{i} Y_{i}
$$

where $N$ is the total number of SUs who participate in the cooperative frequency sensing; $Y_{i}$ is the normalized energy value detected by the $i$ th SU; $W_{i}$ is the $i$ th weight. The test statistic $Y_{w}$ follows the chi-square distribution, and can be expressed as

$$
Y_{w}=\left\{\begin{array}{cc}
\chi_{2 N u}^{2} & H_{0} \\
\chi_{2 N u}^{2}\left(2 \gamma_{w}\right) & H_{1}
\end{array}\right.
$$

Here, $\gamma_{w}=\sum_{i=1}^{N} W_{i} \gamma_{i}$ is the weighted sum of the individual SNRs $\gamma_{i}$ for $i=1,2, \cdots, N$. If all of the weights take a value of unity, the weighted cooperative method is degenerated into the EGC method.

\subsection{Improvement Principle}

For a fixed threshold, the higher SNR of a received signal the higher detection probability of a SU will have. Considering that the distance between the SU and PU is tightly related to a path loss. Particularly, the relation between the average power loss value $P$ and the signal propagation distance $(\mathrm{km})$ can be described by (Choi et al. 2008)

$$
P=10 n \log _{10}(d) \quad(\mathrm{dB})
$$

where $n$ is the power loss exponent which usually takes a constant in the range of 2 4. It is noted that for a sufficient power supply, the coverage of the IEEE802.22 WRAN base station can arrive at $100 \mathrm{~km}$, and the path loss in this range can be up to dozens of dB (Wang et al. 2008). Thus, the effect of the distance on the detection performance is very big.

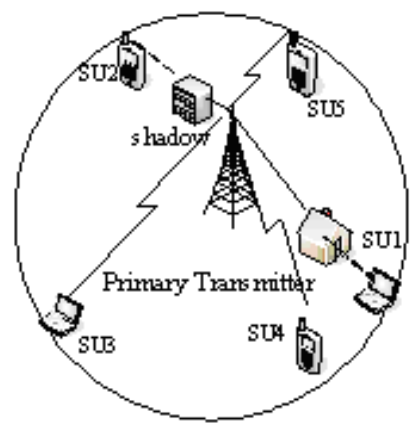

Figure 2. Spectrum sensing scenario

Figure 2 depicts a spectrum sensing scenario for cognitive radio networks, where 5 SUs are detecting the signal transmitted by the PU. In the weighted cooperative spectrum sensing, a reasonable selection of weight coefficients is an effective way to further improve the detection probability. As shown in
Figure 2, the SUs can be divided into two classes as follows.

1) The secondary users SU3, SU4 and SU5 are far from the PU, which leads to bigger path loss. Thus, they should be assigned by smaller distance weighting coefficients. On the other hand, the SNRs of the signals received by these users will be lower. Therefore, these users should be allocated smaller SNR weighting coefficients.

2) The secondary users SU1 and SU2 are closer to the PU, which leads to smaller path loss. Thus, they should be assigned by bigger distance weighting coefficients. However, these two users will have a shadow fading, and thus they will have smaller SNRs. Therefore, they should be assigned by smaller SNR weighting coefficients.

From the above discussion, it is known that on the basis of comprehensively considering the effect of the SNR and distance path loss on the detection performance, a reasonable selection of weighting coefficients can be expected to improve the detection probability further.

This paper presents an improved method for the weighted cooperative spectrum sensing. In this method, a distance weight is introduced, except for the SNR weight. The SNR weight is defined as follows:

$$
W_{\gamma i}=\frac{\gamma_{i}}{\sum_{i=1}^{N} \gamma_{i}}
$$

where $\gamma_{i}$ is the SNR for the $i$ th SU. It implies that if the SNR is smaller its influence on the final decision will be less. On the other hand, the total sum of the SNR weights is 1 .

The distance weight is defined as

$$
W_{d i}=\frac{1}{(N-1)}\left[1-\frac{\log _{10}\left(d_{i}\right)}{\sum_{i=1}^{N} \log _{10}\left(d_{i}\right)}\right]
$$

where $d_{i}$ is the distance between the $i$ th SU and the PU. It can be seen that a bigger $d_{i}$ has less influence on the final decision. The total sum of the distance weights also is 1 .

Considering that the total sum of the weights for the EGC method is $N$, the final weight in this paper is designed as

$$
W_{i}=(N / 2)\left(W_{r i}+W_{d i}\right)
$$

whose total sum is also the value of $N$.

\subsection{Probability Description}

According to the method proposed in this paper, the 
basic process of the weighted cooperative spectrum sensing follows that: each secondary user sends the current distance parameter $d_{i}$, normalized energy value $Y_{i}$ and signal-to-noise ratio $\gamma_{i}$ to the central AP; $N$ values of the final weight $W_{i}$ are determined by using (9), (10) and (11); the weighted energy sum $Y_{w}$ is obtained by using (6); finally the energy sum $Y_{w}$ is compared with a decision threshold to decides whether the PU is occupying the licensed frequency band.

Under various wireless environments, the detection probability $\Psi_{d}$ and the false-alarm probability $\Psi_{f}$ for the weighted spectrum sensing can be expressed as follows (Shahid et al. 2008):

\section{1) AWGN}

For the AWGN channel, the detection and falsealarm probabilities are respectively, given by

$$
\left.\Psi_{d}\right|_{A W G N}=p\left\{Y_{w}>\lambda \mid H_{1}\right\}=Q_{N u}\left(\sqrt{2 \gamma_{w}}, \sqrt{\lambda}\right)
$$

and

$\Psi_{f}=p\left\{Y_{w}>\lambda \mid H_{0}\right\}=\frac{\Gamma(N u, \lambda / 2)}{\Gamma(N u)}$

In the case of independent identically distributed fading, the detection probability is based on instantaneous SNG. Thus, the detection probability is given by

$$
\left.\Psi_{d}\right|_{\text {fading }}=\int_{0}^{\infty} Q_{N u}(\sqrt{2 x}, \sqrt{\lambda}) f_{\gamma_{w}}(x) d x
$$

where $f_{\gamma_{w}}(\cdot)$ is the probability density function of the weighted sum of SNRs. Because the false-alarm probability is independent of SNRs, the false-alarm probability for fading case is the same as that of the AWGN case.

2) Rayleigh Fading

For the Rayleigh fading channel, the probability density function is

$$
f_{\gamma_{w}}(x)=\frac{x^{N-1}}{\Gamma(N) \bar{\gamma}_{w}^{N}} \exp \left(-\frac{x}{\bar{\gamma}_{w}}\right)
$$

where $\bar{\gamma}_{w}$ is determined by

$$
\bar{\gamma}_{w}=\frac{\gamma_{w}}{N}=\frac{1}{N} \sum_{i=1}^{N} W_{i} \gamma_{i}
$$

\section{3) Nakagami Fading}

For the Nakagami fading channel, the probability density function can be expressed as

$$
f_{\gamma_{w}}(x)=\frac{x^{N m-1}}{\Gamma(N m)}\left(\frac{m}{\bar{\gamma}_{w}}\right)^{N m} \exp \left(-\frac{m}{\bar{\gamma}_{w}} x\right)
$$

where $m$ is the Nakagami parameter.

\section{SIMULATION EXPERIMENT}

With Matlab as the platform for simulating the algorithm presented by this paper, we perform the simulation experiment on the single-node energy detection, the EGC detection and the detection presented by this paper under the AWGN, Rayleigh and Nakagami channels.

As shown in Figure 2, the number of users participating in the cooperative spectrum sensing is $N=5$, where SU1 and SU2 suffer from shadow fading. Assume that the value of SNR for each SU can be randomly selected in a range of $-5 \sim 10 \mathrm{~dB}$; the distance between each SU and the PU can be randomly set within $100 \mathrm{~km}$ range. Furthermore, a set of randomly selected simulation parameters with their derived parameters are shown in Table 1. In this table, randomly selected five values of the SNR parameter $\gamma_{i}$ are sequentially assigned to each SU; the derived out parameter $\gamma_{e}$ is the weighted SNR sum for EGC method, whose average value is given by $\bar{\gamma}_{e}=\gamma_{e} / N$. In the simulation, the time-bandwidth product is set as $u=3$. For reasonably comparing the detection performance of different sensing methods, the SNR for the single-node energy detection is intentionally selected as a maximum value, that is, $\gamma_{\max }=9.3924 \mathrm{~dB}$.

Table 1. Parameters and derived parameters for simulation.

\begin{tabular}{|c|c|c|c|c|c|}
\hline $\mathrm{SU}$ & $\gamma_{i}(\mathrm{~dB})$ & $d_{i}(\mathrm{~km})$ & $W_{\gamma i}$ & $W_{d i}$ & $W_{i}$ \\
\hline 1 & -2.8717 & 74.3132 & 0.0380 & 0.1943 & 0.5807 \\
\hline 2 & -1.3412 & 17.1187 & 0.0540 & 0.2133 & 0.6683 \\
\hline 3 & 1.7360 & 75.7740 & 0.1098 & 0.1940 & 0.7595 \\
\hline 4 & 3.3264 & 65.5478 & 0.1583 & 0.1959 & 0.8855 \\
\hline 5 & 9.3924 & 39.2227 & 0.6399 & 0.2025 & 2.1061 \\
\hline \multirow{2}{*}{ SU } & $\gamma_{e}(\mathrm{~dB})$ & $\gamma_{w}(\mathrm{~dB})$ & $\bar{\gamma}_{e}(\mathrm{~dB})$ & $\bar{\gamma}_{w}(\mathrm{~dB})$ & \\
\cline { 2 - 6 } & 11.3313 & 13.4516 & 4.3416 & 6.4619 & \\
\hline
\end{tabular}

In this paper, the detection performance is evaluated by the curves of complementary Receiver Operating Characteristic (abbreviated as ROC), whose horizontal axis represents the false-alarm probability $Q_{f}$ and vertical axis describes the missed-detection probability $Q_{m}$.

For the AWGN channel, a set of complementary ROC curves for three detection methods are shown in Figure 3. This figure shows that the method presented in this paper has the best detection performance. For example, when the false-alarm probability takes a value of 0.01 , the misseddetection probability for the presented method is 0.125 , but the EGC and single-node methods have higher values of 0.48 and 0.39 , respectively. Comparing with the EGC method, the detection 
probability for the presented method is increased by $68.3 \%$.

Figure 4 shows the detection performance for the Rayleigh fading channel, which verifies that the presented method has the best detection performance. Taking a fixed value of 0.01 for the false-alarm probability, it can be figured out that the detection probability of the presented method is increased by $53.1 \%$, comparing with the EGC method.

Figure 5 indicates the detection performance for the Nakagami fading channel with a Nakagami parameter $m=3$, which also shows that the presented method has the best detection performance. For the convenience of comparison, a value of the false-alarm probability is still taken as 0.01 . It can be verified that the detection probability of the presented method is increased by $53.2 \%$, comparing with the EGC method.

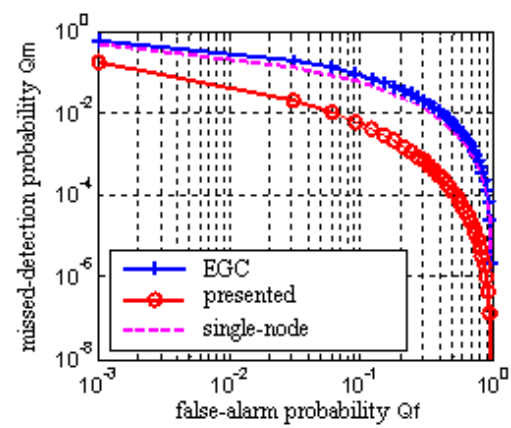

Figure 3. Complementary ROC for AWGN channel

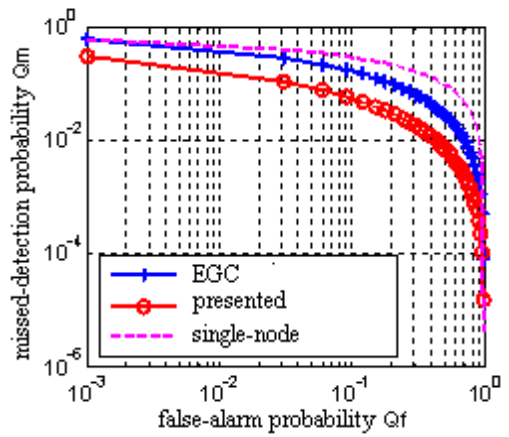

Figure 4. Complementary ROC for Rayleigh channel

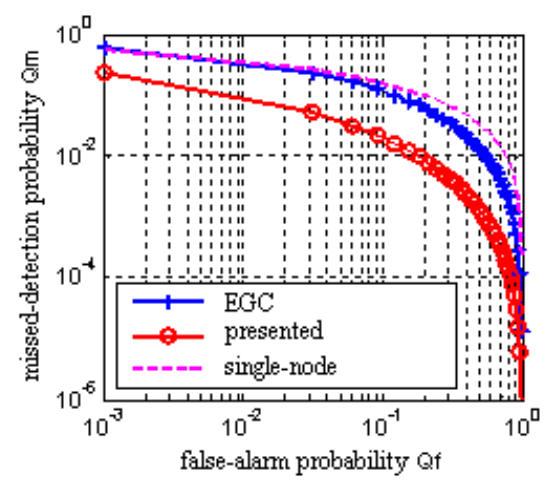

Figure 5. Complementary ROC for Nakagami channel

\section{CONCLUSION}

The method presented by this paper for the weighted cooperative frequency sensing has comprehensively considered the effects of the signal-to-noise ratios and the path losses on the detection performance. The designed weighting formulas can reasonably fuse the signal-to-noise ratios and distance parameters, which provide a good mathematical model for improving the detection performance. Using randomly produced simulation parameters, the simulation results for the AWGN, Rayleigh and Nakagami fading channels show that the presented method has the best detection performance, comparing with the single-node and EGC methods.

\section{REFERENCES}

[1] Choi, Hyun-Ho et al. 2008. Adaptive sensing threshold control based on transmission power in cognitive radio systems. Third International Conference on Cognitive Radio Oriented Wireless Networks and Communications (1):1-6.

[2] Digham, E. F. et al. 2003. On the energy detection of unknown signals over fading channels. IEEE International Conference on Communications (5):3575 -3579.

[3] Ghasemi, A \& Sousa, E. S. 2005. Collaborative spectrum sensing for opportunistic access in fading environments. IEEE DYSPAN: 131-136.

[4] Lin, Ying-pei et al. 2010. A spectrum sensing method based on high order cyclic statistic. Journal of Shanghai Jiaotong University 44(2):209-213.

[5] Luo, Tao et al. 2009. Technology of Multiuser collaborative communications and spectrum sensing. ZTE Communications 15(2):60-64.

[6] Pan, Jian-guo \& Zai, Xu-ping. 2009. Spectrum sensing in cognitive radio based on energy detection. Journal of Shanghai University 15(1):54-59.

[7] Shahid, M. I. B. \& Kamruzzaman, J. 2008. Weighted soft decision for cooperative sensing in cognitive radio networks. the 16th IEEE International Conference (1):1-6.

[8] Urkowitz, H. 1967. Energy detection of unknown deterministic signals. Proceedings of the IEEE 55(4):523531.

[9] Visser, F. E. et al. 2008. Multinode spectrum sensing based on energy detection for dynamic spectrum access. Vehicular Technology Conference 5:1394-1398.

[10] Wang, Jian-ping et al. 2008. Cognitive Radio. Beijing: National Defence Industry Press.

[11] Zhou, Xiao-fei \& Zhang, Hong-gang. 2007. Cognitive Radio Principles. Beijing: Beijing University of Posts and Telecommunications Press. 